\title{
Effects of Transverse Magnetic Field with Different Alternating Currents on Heat Flux Density Distributions of Plasma Arc
}

\author{
Xiaojuan Dong, Jianbing Meng*, Xiuting Wei and Zhanmin Yin
}

\author{
School of Mechanical Engineering, Shandong University of Technology, Zibo 255049, China
}

\begin{abstract}
An external transverse-alternating magnetic field with sinusoidal and triangular alternating currents was applied to a combined plasma arc to create a plasma arc for expanding the cross section of arc column and flatting the distributions of arc temperature. Two mathematical models were developed to describe the heat flux density distributions of the combined plasma arc driven by a transverse-alternating magnetic field with sinusoidal and triangular alternating currents. The behavior of plasma arc under the external transverse-alternating magnetic field imposed perpendicular to the plasma current was discussed theoretically and experimentally by changing various parameters such as working gas flux, arc current, magnetic flux density including its wave form and the standoff from the nozzle to the workpiece. The analytical results show that it is feasible to adjust the shape and heat flux density of the combined plasma arc by the transverse- alternating magnetic field, which expands the region of combined plasma arc thermal treatment and uniforms the heat flux density upon the workpiece. Changing the waveform of the alternating current can also control the heat flux density distribution. As well as, calculated heat flux density distributions of combined plasma arc driven by the external transverse-alternating magnetic field show a good agreement with experimental data. The magnetic field with triangular alternating current can flat the heat flux density distribution on the anode rather than sinusoidal one. This approach to flat the heat flux density distribution on the anode surface will give an effective controllability to the combined plasma arc application.
\end{abstract}

Keywords: Alternating current, combined plasma arc, heat flux, transverse magnetic field.

\section{INTRODUCTION}

Plasma arc has generally been used for material processing such as welding, cutting, spraying, etc. Its characteristics have a crucial and direct influence on above processing quality. Usually, the energy distribution of the plasma arc is limited in a smaller region and the temperature gradient of the plasma arc exerts more variation in the arc column owing to the avalanche effect of gas discharge and the constraint of plasma arc by its own magnetic field. The above features of a plasma arc, plasma welding and plasma cutting are better than other corresponding processes. However, some material processes, including plasma arc cleaning [1] and surface modification using plasma arc [2], don't desire the heat source to be concentrated, but require a uniform and controllable plasma arc. Therefore, conventional plasma arc is not a convenient tool for heating over a wide area because of the concentrated heat flux in a small arc root. Unfortunately, many studies on the jet characteristics of transferred plasma arc and non-transferred plasma arc have been published, however, little attentions are given to a numerical simulation of the combined plasma arc [3-5]. Furthermore, as an ideal heat source, combined plasma arc has been generally applied to precise welding and also involves complex physical phenomena [6].

\footnotetext{
*Address correspondence to this author at the School of Mechanical Engineering, Shandong University of Technology, Zibo 255049, China;

Tel: +86-0533-2786909; E-mail: jianbingmeng@126.com
}

In order to obtain a shape-controlled soft plasma arc, some researchers attempted to introduce an external magnetic field to expand the arc column' cross section and flatten the distributions of arc current and temperature, considering the electromagnetic property of the plasma arc. J. Awrejcewicz combined analytical and numerical analyses of oscillations in a string-type generator [7, 8]. Meanwhile, they found that the form of plasma arc in the electromechanical system showed some interesting nonlinear phenomena [9]. D.B. Sobyanin et al. carried out laboratory experiments to investigate the evolution of the plasma jet injected transverse to the magnetic field and observed that the plasma jet propagation existed in two stages [10]. In the first stage, the magnetic field expulsion was detected. At the second stage, deceleration and deflection of the plasma jet due to field-aligned currents were investigated. A. Zhainakov et al. performed a numerical simulation of an open electric arc in a transverse magnetic field within the three-dimensional mathematical model. Furthermore, the effect of external controlled discharge parameters and the magnetic field of the electrodes on the characteristics of a plasma arc was also revealed [11]. The results showed that the angle of deviation of an arc in space was directly proportional to the interelectrode spacing and inversely proportional to the current strength. R. Akiho et al. succeeded in expanding the arc area by imposing an alternating magnetic field perpendicularly to the arc current [12]. The arc was driven by electromagnetic force resulted from the interaction between the imposed magnetic field and the arc current. Furthermore, R. Akiho et al. investigated the 
movement of the magnetically driven arc under the swirling motion of the plasma gas flow. Considering the Magnus effect, the theoretical model for the arc motion was constructed. Then numerical calculations were conducted, and results from experimental observations showed that the theoretical modeling was reasonable.

Up to now, many studies on the characteristics of a plasma arc under an external transverse DC magnetic field have been published. However, little attentions are given to the investigations on the oscillating motion and heat flux distribution of plasma arc under an external transverse alternating magnetic field. In previous studies, W.J. Xu et al. carried out some experiments in flexible forming of sheet metal using the combined plasma arc under an external magnetic field [13]. Meanwhile, the effects of the combined plasma arc in the external magnetic field on the metal surface quality and efficiency of flexible forming were analyzed. The experimental results show that it is feasible to improve the flexible forming of sheet metal using the plasma arc with the external transverse AC magnetic field. However, the heat flux distribution of the combined plasma arc is to vary and the conventional Gauss model of the heat flux density of the combined plasma arc is inappropriate owing to the effect of the external transverse alternating magnetic field. Consequently, it has to depend on experimental measurement to obtain the heat flux distributions of plasma arc, which limits the application of the plasma arc with external transverse alternating magnetic field. The authors have developed a combined plasma arc system to investigate the characteristics of combined plasma arc injected transverse to a transverse-alternating magnetic field with a triangular alternating current [14]. The characteristic of combined plasma arc under the external transversealternating magnetic field imposed perpendicular to the plasma current was discussed. The heat pattern on the workpiece surface was adjustable by the external transverse alternating magnetic field. However, few studies have addressed the effect of different alternating currents on the heat flux density distribution.

In this paper, an external transverse-alternating magnetic field with sinusoidal and triangular alternating currents was applied to a combined plasma arc to create a soft and uniform arc for expanding the heated area of metal surface. This study attempts to investigate the heat flux density distribution of the combined plasma arc driven by external transverse-alternating magnetic field both theoretically and experimentally. By analyzing the principle of the external magnetic field driving the combined plasma arc, two mathematical models are developed to describe the heat flux density distribution of combined plasma arc on the treated surface driven by the external transverse-alternating magnetic field with sinusoidal and triangular alternating currents. Moreover, the influence of processing parameters, such as magnetic flux density, working current, argon flow rate and the distance from the nozzle outlet to the workpiece anode on the heat flux density distribution of the combined plasma arc is also investigated. And the effect of the alternating current type of magnetic field on the plasma behavior has been clarified.

\section{FOUNDATIONAL PRINCIPLE}

Plasma makes up of a large number of charged particles. The motion of charged particles can change the electromagnetic field. In turn, the electromagnetic field changes can influence the motion of particles. When the interaction between charged particles is neglected, and the electromagnetic field generated by the motion of charged particles is much smaller than the external electromagnetic field, plasma can be approximated as a set composed of a large amount of independent charged particles.

The motion of charged particles in uniform magnetic field can be expressed as

$m \frac{d \mathbf{v}}{d t}=q(\mathbf{v} \times \mathbf{B})$

where, $m, t$ and $q$ is the mass $(\mathrm{kg})$, time (s), electric quantity (C), respectively. $\mathbf{V}$ is the velocity vector of plasma in the magnetic field. B is the magnetic flux density of external transverse alternating magnetic field.

When $\mathrm{Z}$ direction is selected as the applied direction of magnetic field, Eq. (1) can be expressed as

$m \mathbf{e} \cdot \frac{d \mathbf{v}}{d t}=m \frac{d v_{/ /}}{d t}=q(\mathbf{v} \times \mathbf{B}) \cdot \frac{\mathbf{B}}{B}=0$

where, $\mathbf{e}$ is the unit vector of $\mathrm{Z}$ direction. $v_{/ /}$is the velocity component of plasma parallel to the external magnetic field direction.

Similarly, the inner product of Eq. (1) and the velocity vector $\mathbf{V}$ can be expressed as

$\frac{d}{d t}\left(\frac{m \mathbf{v}^{2}}{2}\right)=q(\mathbf{v} \times \mathbf{B}) \cdot \mathbf{v}=0$

Combined the law of conservation of energy with Eq. (2) and Eq. (3), Eq. (1) can be expressed as

$$
\left\{\begin{array}{l}
m d v_{x} / d t=q v_{y} B \\
m^{d v_{y}} / d t=-q v_{x} B \\
m d v_{z} / d t=0
\end{array}\right.
$$

where, $v_{x}, v_{y}$ and $v_{z}$ is the velocity component of plasma in $\mathrm{X}$, $\mathrm{Y}, \mathrm{Z}$ direction. With the derivative of Eq. (4) with respect to $t$, Eq. (4) can be expressed as

$$
\left\{\begin{array}{l}
\frac{d^{2} v_{x}}{d t}=\frac{q B}{m} \cdot \frac{d v_{y}}{d t}=-\left(\frac{q B}{m}\right)^{2} v_{x} \\
\frac{d^{2} v_{y}}{d t}=-\frac{q B}{m} \cdot \frac{d v_{x}}{d t}=-\left(\frac{q B}{m}\right)^{2} v_{y} \\
m \frac{d^{2} v_{z}}{d t^{2}}=0
\end{array}\right.
$$


The velocity component of plasma in the external magnetic field can be obtained from Eq. (5), and can be expressed as

$$
\left\{\begin{array}{c}
v_{x}=v_{\perp} \cos \left(\omega_{c} t+\delta\right) \\
v_{y}=-v_{\perp} \sin \left(\omega_{c} t+\delta\right) \\
v_{z}=v_{/ /}=\text {const }
\end{array}\right.
$$

where, $v_{\perp}$ is the velocity component of plasma perpendicular to the external magnetic field. $\omega_{c}$ is the rotating angular velocity of plasma in the external magnetic field.

A schematic illustration to expand the combined plasma arc using an external transverse alternating magnetic field (ETAMF) is shown in Fig. (1). The device consists of a combined plasma arc torch with a tungsten cathode, a nozzle anode and an workpiece anode, a DC power supply to generate a combined plasma arc, a gas feeding system for combined plasma arc, a support frame to fix the torch, a excitation device including two excitation coils and two magnetic irons to perpendicularly introduce the magnetic field into the plasma arc, an AC power supply for the external transverse magnetic field, which is connected to the excitation coils, can supply sinusoidal and triangular alternating currents. In this paper, the type and the frequency of AC power supply are sinusoidal, triangular waves and $50 \mathrm{~Hz}$, respectively. Assuming the movement time of combined plasma from the nozzle outlet to the surface of the workpiece is $T$, when the excitation frequency of the alternating magnetic field $f$ less than $1 / 2 \mathrm{~T}$, the oscillating amplitude and heat flux density distribution of combined plasma arc are independent of the excitation frequency $[3$, $14]$.

When the DC power supply is applied between the cathode and anode, the gas is to be ignited and the combined plasma arc is to be generated. Then the AC power supply is applied between two excitation coils, the alternating magnetic field is to be generated, which is perpendicularly introduced into the above combined plasma arc. As a result, the combined plasma arc oscillates by the alternating electromagnetic force. It is well known that plasma arc consists of a lot of charged particles and each charged particle is driven by the Lorentz force under the external transverse magnetic field. Consequently, charged particles are in gyroscopic motion neglecting the interaction and the self-induced magnetic field of these particles. The gyration radius of charged particles can be obtained from Eq. (6), and expressed as

$r=\frac{m v}{q B}$

The combined plasma arc is also in the oscillatory motion along the transverse direction, since plasma being made up of all charged particles. Furthermore, the form of the arc column is like a bell for the cause of excitation frequency and thermal inertia of the combined plasma arc.

\section{MODELLING APPROCH}

\subsection{Heat Flux Density of Plasma Arc under ETAMF with Sinusoidal Alternating Current}

In order to establish the mathematical model of arc heat flux distribution upon the anode, the assumptions are summarized as follows: The total heat flux $W(\mathrm{~W})$ of the

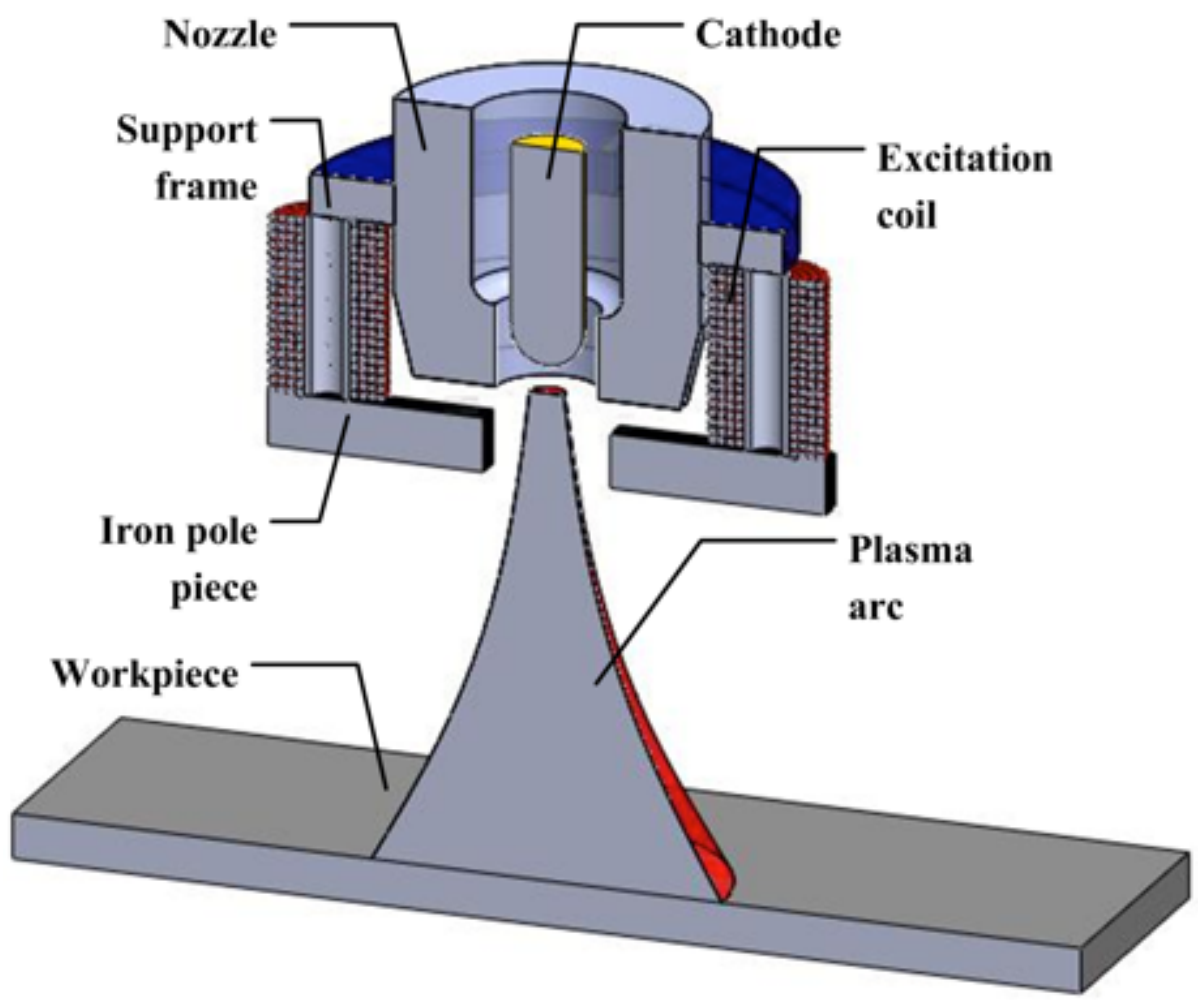

Fig. (1). Generator device of plasma arc with an ETAMF. 
plasma arc is transferred to the anode, neglecting the heating effect of viscous dissipation; Without the external alternating magnetic field, the root of combined plasma arc can be regarded as a round area heat source with the radius of $R_{0}$ [13] ; Under the external transverse alternating magnetic field, the root of combined plasma arc can be considered as an elliptic area heat source with the shorter axis of $R_{0}$ and the longer axis of $\left(R_{0}+l_{0}\right)$.

Driven by the external transverse alternating magnetic field, the heat flux density $h\left(\mathrm{~W} / \mathrm{m}^{2}\right)$ of plasma arc can be written as

$h=\frac{W}{\pi R_{0}^{2}}$

Supposing that the distance from any point in the arc root to the center of plasma arc is $R$, heat input into the small element of the arc root $(d S)$ for the time $(d t)$ can be expressed as

$d Q=h\left[\pi R_{0}(R+d R)-\pi R_{0} R\right] d t$

Because alternating current is sinusoidal current type, the applied alternating field can be expressed as

$B(t)=B_{0} \sin \omega t$

where, $B_{0}$ is the maximum magnetic flux density (T), $\omega$ is the angular velocity.

The displacement of any point $R$ in the root of combined plasma arc can be expressed as

$x=l_{0} \sin \omega t+R$

where, $l_{0}$ is the maximum oscillating amplitude of combined plasma arc in the external magnetic field. It can be obtained from [13].

$l_{0}=\frac{60 I B_{0} D^{2}}{\rho Q v}$

Combined Eq. (8) with Eq. (9) and the derivative of Eq. (11) with respect to $t$, the total heat input the anode can be expressed as

$Q=\iint \frac{W}{\omega R_{0}} \cdot \frac{1}{\sqrt{l_{0}^{2}-(x-R)^{2}}} d R \cdot d x$

During the half period of arc oscillatory motion, $W^{\prime}$ is defined as the heat input into the workpiece anode in unit time, which can be written as

$W^{\prime}=\frac{\omega}{\pi} Q$

The heat flux density $H$ can be expressed as

$H=\frac{W^{\prime}}{S}=\frac{d W^{\prime}}{\pi R_{0}(x+d x)-\pi R_{0} x}$

With the simultaneous solution of Eq. (13), Eq. (14) and Eq. (15), the heat flux density $H$ can be written as
$H=\int \frac{W}{\left(\pi R_{0}\right)^{2}} \cdot \frac{1}{\sqrt{l_{0}^{2}-(x-R)^{2}}} d R$

During a half cycle of the magnetic field, the root centre of combined plasma arc moves from $-l_{0}$ to $l_{0}$. For $l_{0}+R_{0} \leq x \leq l_{0}-R_{0}$, the whole arc root passes point $x$. However, for $x<l_{0}+R_{0}$ or $x>l_{0}-R_{0}$, a part of the root of combined plasma arc passes the point. Consequently, the heat flux density can be transferred as

$$
H(x)=\left\{\begin{array}{lr}
\frac{W}{\left(\pi R_{0}\right)^{2}}\left(\frac{\pi}{2}+\arcsin \left(\frac{R_{0}+x}{l_{0}}\right)\right) & -l_{0}-R_{0}<x<-l_{0}+R_{0} \\
\frac{W}{\left(\pi R_{0}\right)^{2}}\left(\arcsin \left(\frac{R_{0}-x}{l_{0}}\right)+\arcsin \left(\frac{R_{0}+x}{l_{0}}\right)\right) & -l_{0}+R_{0} \leq x \leq l_{0}-R_{0} \\
\frac{W}{\left(\pi R_{0}\right)^{2}}\left(\frac{\pi}{2}+\arcsin \left(\frac{R_{0}-x}{l_{0}}\right)\right) & l_{0}-R_{0}<x<l_{0}+R_{0}
\end{array}\right.
$$

\subsection{Heat Flux Density of Plasma Arc under ETAMF with Triangular Alternating Current}

Similarly, if an external magnetic field with triangular alternating current is applied perpendicularly to the combined plasma arc, the heat flux density distribution of combined plasma arc on the anode can be obtained from the previous published article [13], and be expressed as

$$
H(x)=\left\{\begin{array}{cc}
\frac{W}{2 \pi R_{0}^{2}} \cdot \frac{l_{0}+x+R_{0}}{l_{0}} & \left(-l_{0}-R_{0}<x<-l_{0}+R_{0}\right) \\
\frac{W}{2 \pi R_{0}^{2}} \cdot \frac{2 R_{0}}{l_{0}} & \left(-l_{0}+R_{0} \leq x \leq l_{0}-R_{0}\right) \\
\frac{W}{2 \pi R_{0}^{2}} \cdot \frac{l_{0}-x+R_{0}}{l_{0}} & \left(l_{0}-R_{0}<x<l_{0}+R_{0}\right)
\end{array}\right.
$$

\section{CALCULATIONS AND DISCUSSIONS}

As shown in Fig. (1), the whole system can be divided into the part for generating the plasma arc and another part for generating the external transverse triangular wave alternating magnetic field. The working parameters in each part are shown in Tables $\mathbf{1}$ and $\mathbf{2}$, respectively. In the Table 2, $I_{0}, N, \Phi, L$ and $f$ are the excitation current, coil turn, coil diameter, coil length and excitation frequency, respectively. With above working parameters and measured variables, the models on the oscillating amplitude and the heat flux density distribution of plasma arc are solved. As a result, the effect of process parameters, such as the gas flow rate $Q$, the arc current $I$ and the overhang from the nozzle outlet to the anode workpiece $D$ for above two models are obtained.

Table 1. Parameters of the generator device of plasma arc.

\begin{tabular}{|c|c|c|c|c|c|c|c|}
\hline \multicolumn{7}{|c|}{ Parameters of Plasma Arc } \\
\hline \hline$I(\mathrm{~A})$ & 15 & 20 & 25 & 15 & 15 & 15 & 15 \\
\hline$Q(\mathrm{~L} / \mathrm{min})$ & 5 & 5 & 5 & 4 & 6 & 5 & 5 \\
\hline$D(\mathrm{~mm})$ & 7 & 7 & 7 & 7 & 7 & 6 & 8 \\
\hline$v(\mathrm{~m} / \mathrm{s})$ & 12.5 & 25.8 & 36.3 & 8.95 & 13.3 & 11.9 & 13.2 \\
\hline
\end{tabular}


Table 2. Parameters of the external transverse alternating magnetic field.

\begin{tabular}{|c|c|c|c|c|}
\hline \multicolumn{5}{|c|}{ Parameters of ETAMF } \\
\hline \hline$I_{0}(\mathrm{~A})$ & $N$ & $\Phi(\mathrm{mm})$ & $L(\mathrm{~mm})$ & $f(\mathrm{~Hz})$ \\
\hline $0 \sim 1.2$ & 200 & 44 & 38 & 50 \\
\hline
\end{tabular}

\subsection{Effects of the Gas Flow Rate on Heat Flux Density Distributions}

Under typical operation conditions $(I=15 \mathrm{~A}, D=7 \mathrm{~mm}$, $B=15 \mathrm{mT}$ ), heat flux density distributions of plasma arc on the anode workpiece surface are calculated in this section by importing three different argon gas flow rates: $4,5,6 \mathrm{~L} / \mathrm{min}$. Fig. (2a, b), respectively, show theoretical distributions of heat flux density on the anode for sinusoidal and triangular alternating currents. As shown in Fig. (2a, b), the radius of heat flux distribution decreases with the increase of gas flow rate. It also can be seen that the heat flux density at the center of arc root ascends and the gradient of heat flux
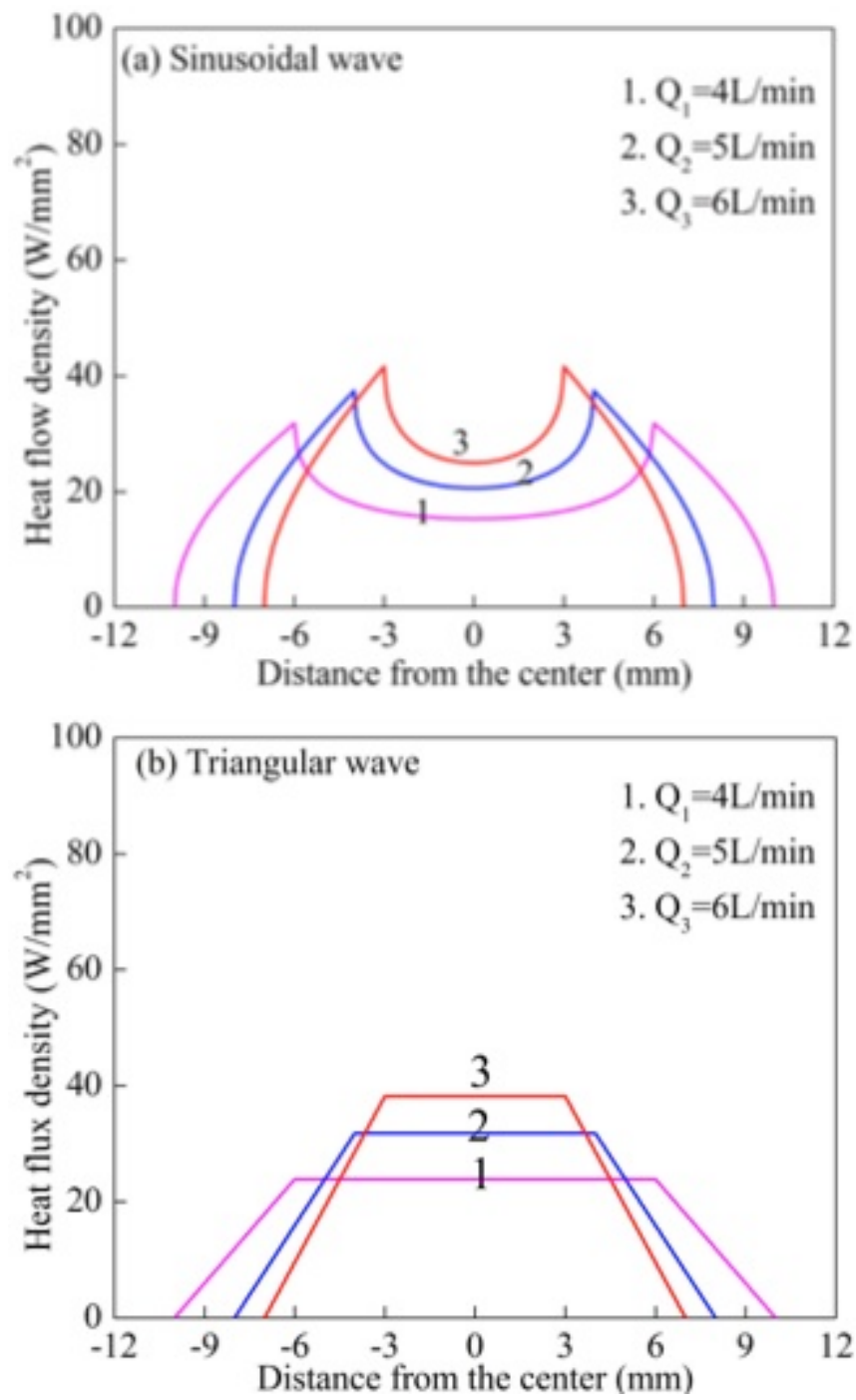

Fig. (2). Effect of the gas flow rate on the heat flux density distribution in different alternating currents. density near the center of arc root increases with the increase of gas flow rate. The reason is that increasing the gas flow rate is to accelerate the velocity of plasma on the nozzle outlet and to reduce the oscillating amplitude of plasma on the anode workpiece. On the other hand, due to the effect of stabilizing arc arising from the increase of plasma velocity, it is difficult to flatten the heat flux density of the plasma arc. As a result, it can be concluded that the heat flux of combined plasma arc on the workpiece surface is more concentrated with the increase of gas flow rate.

\subsection{Effects of the Arc Current on Heat Flux Density Distributions}

Under typical operation conditions $(Q=5 \mathrm{~L} / \mathrm{min}, D=7$ $\mathrm{mm}, B=15 \mathrm{mT}$ ), heat flux density distributions of combined plasma arc on the anode workpiece surface are calculated in this section by importing three different arc currents: 15, 20 and 25 A. Fig. (3a, b), respectively, show theoretical distributions of heat flux density on the anode for sinusoidal and triangular alternating currents. As shown in Fig. (3a, b), the radius of heat flux distribution decreases with the increase of arc current. It can be also seen that the heat flux
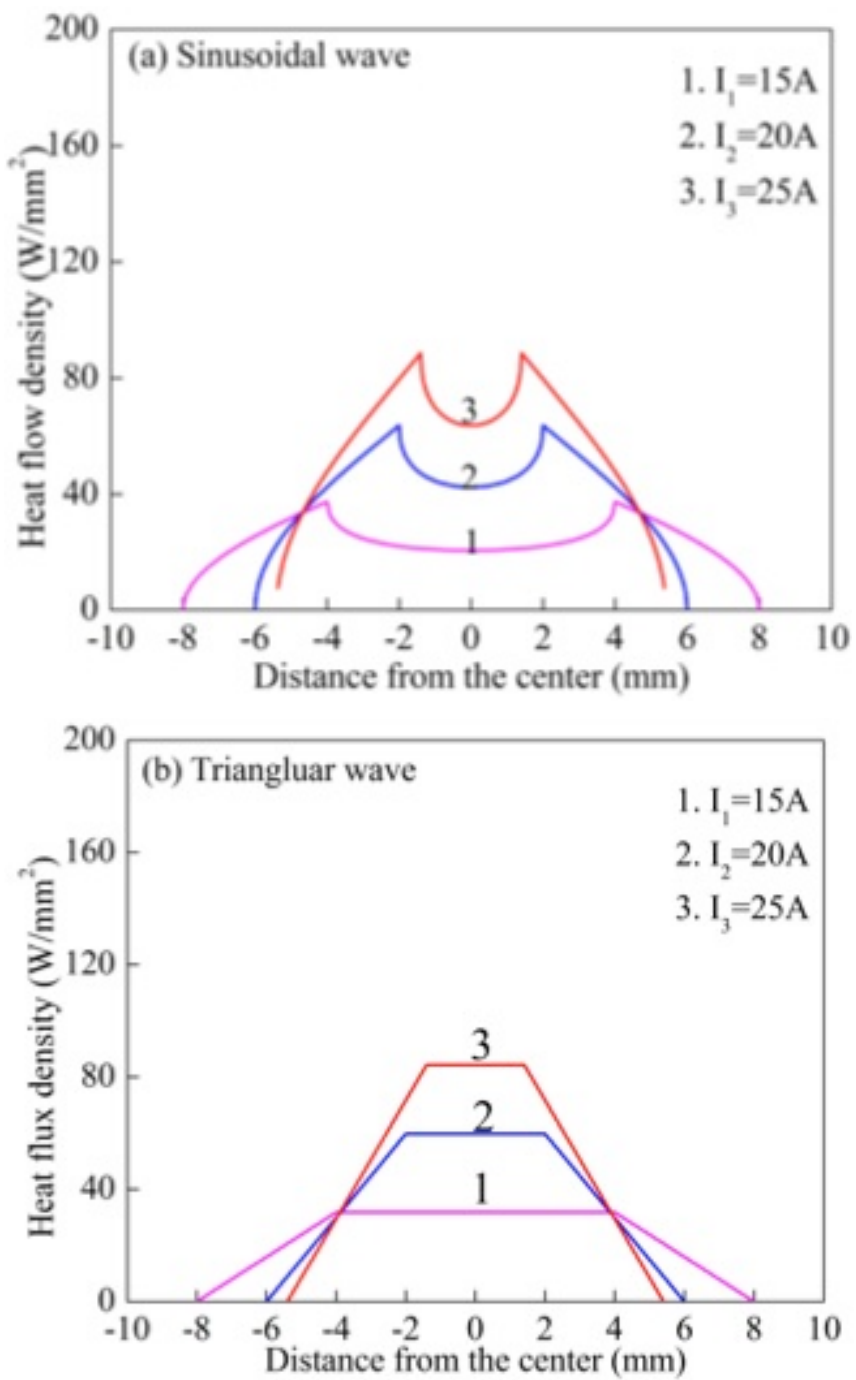

Fig. (3). Effect of the arc current on the heat flux density distribution in different alternating currents. 
density at the center of arc root ascends and the gradient of heat flux density near the center of arc root increases with the increase of arc current. The reason is that increasing the arc current is to decrease the oscillating amplitude of plasma arc in the external transverse alternating magnetic field, which leads to the shrink of heat flux density distribution. On the other hand, the constraint by arc own magnetic field is strengthened and the heat flux of the combined plasma arc is more concentrated with the increase of arc current. Consequently, it can be concluded that it is more difficult to expand the distribution region and to flatten the heat flux density of combined plasma arc on the anode workpiece surface.

\subsection{Effects of the Nozzle Overhang on Heat Flux Density Distributions}

Under typical operation conditions ( $I=15 \mathrm{~A}, Q=5 \mathrm{~L} / \mathrm{min}$, $B=15 \mathrm{mT}$ ), heat flux density distributions of combined plasma arc on the anode workpiece surface are calculated in this section by importing three different overhangs: 6,7 and $8 \mathrm{~mm}$. Fig. (4a, b), respectively, show theoretical distributions
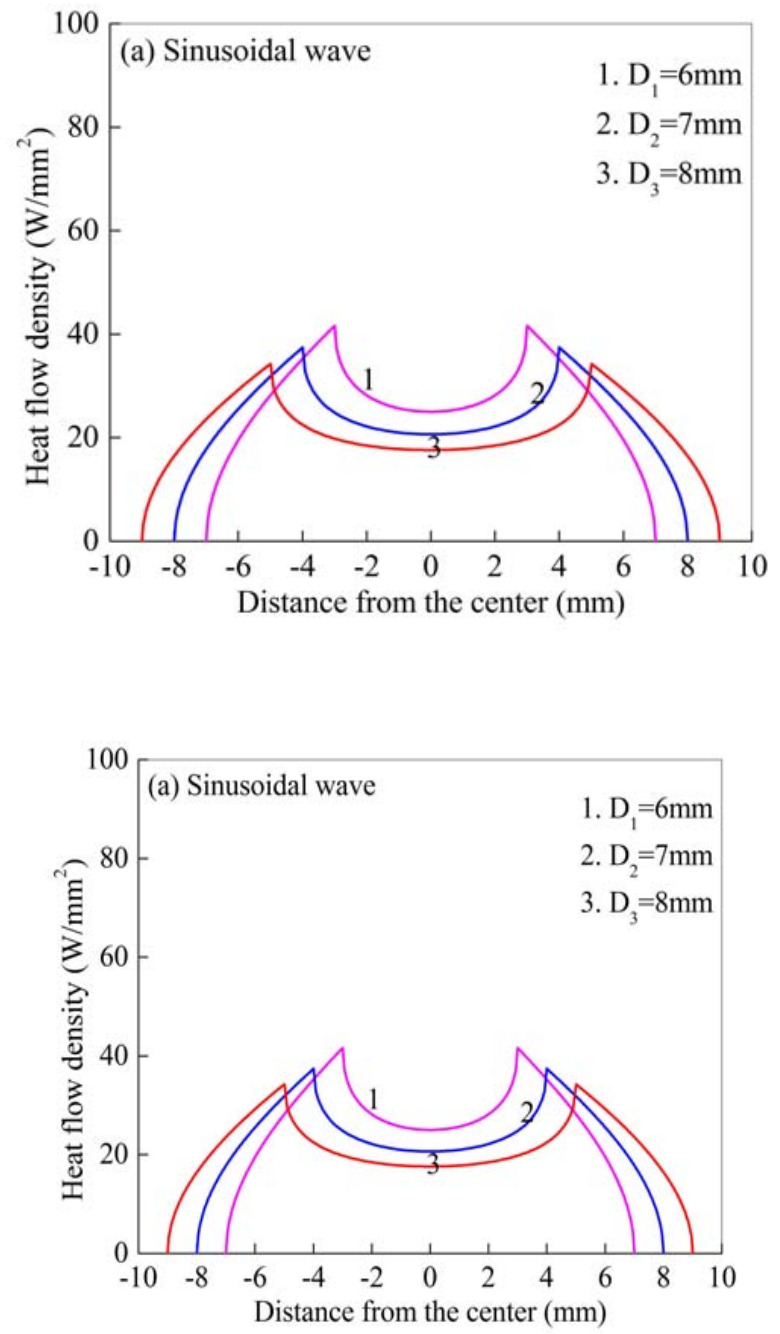

Fig. (4). Effect of the nozzle overhang on the heat flux density distribution in different alternating currents. of heat flux density on the anode for sinusoidal and triangular alternating currents. As shown in Fig. (4a, b), the radius of heat flux distribution increases with the expansion of overhang. It also can be seen that the heat flux density at the center of arc root descend and the gradient of heat flux density near the center of arc root reduces with the increase of the overhang from the nozzle outlet to the anode workpiece. The reason is that increasing the overhang means to expand the oscillating amplitude and to improve the heat flux distribution radius. Meanwhile, with the expansion of the heating area, the heat flux on the center of arc root is dispersed into the whole heating region. As a result, it is much easier to flatten the heat flux density of plasma arc on the workpiece surface with the increase of the overhang from the nozzle outlet to the anode workpiece.

\subsection{Effects of the Magnetic Flux Density on Heat Flux Density Distributions}

Under typical operation conditions $(I=15 \mathrm{~A}, Q=5 \mathrm{~L} / \mathrm{min}$, $D=7 \mathrm{~mm}$ ), heat flux density distributions of plasma arc on the anode workpiece surface are calculated in this section by importing three different magnetic flux density: 10, 12.5 and 15 mT. Fig. (5a, b), respectively, show theoretical
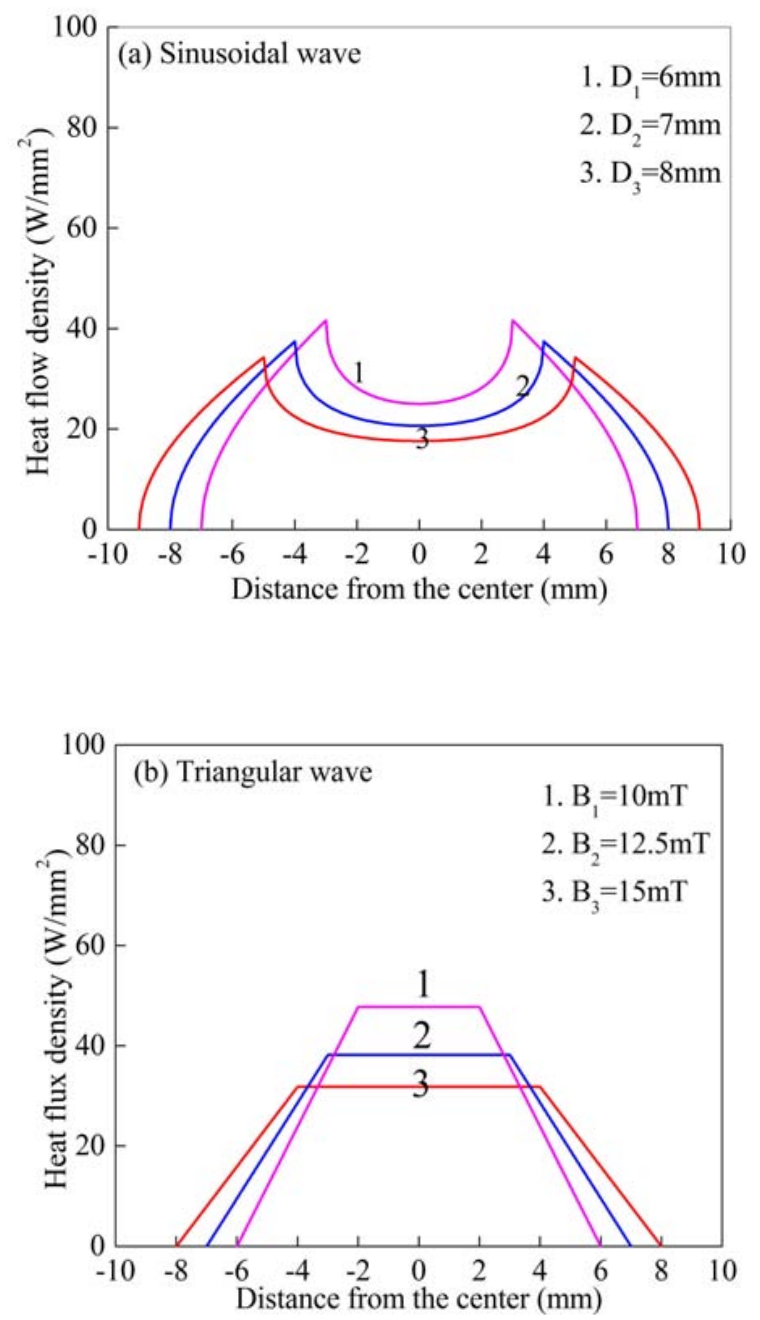

Fig. (5). Effect of the magnetic flux density on the heat flux density distribution in different alternating currents. 
distributions of heat flux density on the anode for sinusoidal and triangular alternating currents. As shown in Fig. (5a, b), the radius of heat flux distribution increases with the enhancement of magnetic flux density. It also can be seen that increasing the magnetic flux density is to decrease the heat flux density at the center of the arc root. Furthermore, the gradient of heat flux density near the center of arc root is descended with the increase of magnetic flux density. The reason is that increasing magnetic flux density is to increase the oscillating amplitude of the plasma arc. Moreover, the extension of the heat flux distribution radius means more heat flux on the center of arc root is transferred into the whole heating area. Consequently, the heat flux density of plasma arc on the anode workpiece is easier flattened for the increase of magnetic flux density.

\subsection{Effects of the Alternating Current Type on Heat Flux Density Distributions}

As shown in Figs. (2-5), heat flux density distributions of plasma arc driven by the external transverse sinusoidal wave magnetic field is obviously different from the ones under the triangular wave magnetic field. Compared Figs. (2a, 3a, 4a, 5a) with Figs. (2b, 3b, 4b, 5b), it can be found that heat flux density gradient of combined plasma arc with the sinusoidal excitation waveform is less than the one of combined plasma arc with the triangular waveform when the processing parameter is the same. That is to say, the former is more uniform than the latter in the near edge of anode surface. On the contrary, near the center of combined plasma arc distributions on the anode surface, the heat flux density gradient of combined plasma arc with the sinusoidal excitation waveform is more than the one of combined plasma arc with the triangular waveform. That is, the latter is more flat than the former in the center of plasma arc distributions.

\subsection{Experimental Verification}

In this experiment, the applied magnetic flux density is a triangular wave, the process parameters such as the argon gas flow rate, the arc current and the overhang from the nozzle to the anode workpiece are $5 \mathrm{~L} / \mathrm{min}, 15 \mathrm{~A}$ and $6 \mathrm{~mm}$, respectively. Fig. (6a) show the shape of the oscillating combined plasma arc without magnetic field. From Fig. (6a), it can be seen that the radius of heat flux distribution is $2 \mathrm{~mm}$. With a sinusoidal and a transverse alternating magnetic field $(B=15 \mathrm{mT})$, the plasma arc is driven to oscillate on the anode workpiece. As shown in Fig. $(6 \mathbf{b}, \mathbf{c})$, the radius of heat flux distributions with sinusoidal and triangular alternating currents is $4.7 \mathrm{~mm}$ and $5 \mathrm{~mm}$, respectively. From Fig. (6a-c), the oscillating amplitude of plasma arc can be measured $(l=2.6 \mathrm{~mm}$ and $l=3 \mathrm{~mm})$. It can be found that measured values of the plasma arc oscillating amplitude agree fairly well with the theoretical value $(l=2.8 \mathrm{~mm})$ from Eq. 12 , and the agreement between them is more than $92.8 \%$ and $93 \%$, respectively. Consequently, it is feasible to adjust the shape of the combined plasma arc driven by the external transversealternating magnetic field with the sinusoidal and triangular alternating currents.

Fig. $(\mathbf{7 a}, \mathbf{b})$ show the distributions of heat flux density in no magnetic field, in sinusoidal and triangular currents alternating magnetic field $(B=15 \mathrm{mT})$, respectively. As shown in Fig. (7), without no magnetic field $(B=0)$, the heat flux density on the center of arc root from the model developed in this paper $\left(H=95.5 \mathrm{~W} / \mathrm{mm}^{2}\right)$ accords with the value calculated from Gauss model $\left(H=101.6 \mathrm{~W} / \mathrm{mm}^{2}\right)$. The error between them is less than 6\%. Compared Fig. (7a) with Fig. (7b), it can be found that heat flux density distribution of plasma arc with the sinusoidal excitation current is more uniform than the one of plasma arc with the triangular current when the processing parameter is the same. It also can be seen that with the sinusoidal and triangular external magnetic field $(B=15 \mathrm{mT})$, the heat flux densities and their distribution radiuses from the model developed in this paper are more than calculated values of heat flux density from Gauss model and the model in this paper with no external magnetic field, respectively.

The distributions of the heat flux density on the workpiece surface are measured using a specially designed copper anode. It is $400 \mathrm{~mm}$ long and $200 \mathrm{~mm}$ wide. The anode is divided into several sections through which a cooling-water channel passes. Thermocouples are inserted into the water channel at the inlet and outlet of each section. Heat flow from the arc root into each section is evaluated from the temperature increase of the cooling water through the channel. Fig. (8a, b), respectively, show measured distributions of heat flux density on the anode for a sinusoidal current and the triangular current. Theoretical heat flux density distributions in Fig. $(\mathbf{8 a}, \mathbf{b})$ are calculated under the assumption of $B=15 \mathrm{mT}$. In Fig. (8), solid circles show experimental results of heat flux density, and solid lines represent the predicted relations. The total heat flow is experimentally determined from the difference between
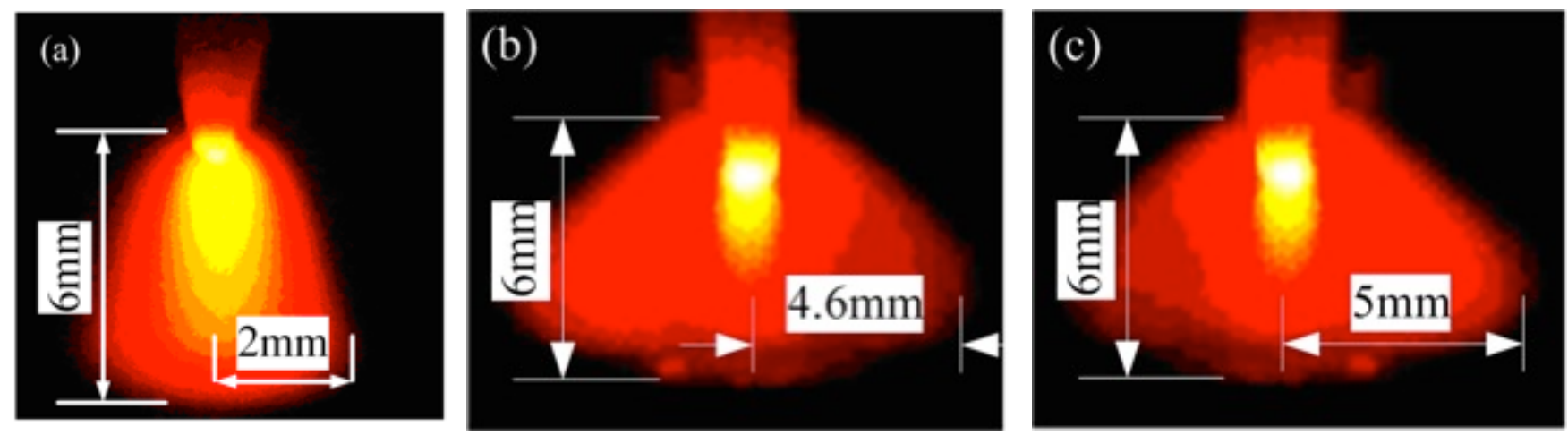

Fig. (6). Distributions of plasma arc forms with or without alternating magnetic field with sinusoidal and triangular alternating currents 
temperature of the water at the inlet and that at the outlet of the anode. The total heat flow into the anode does not vary according to the application of the magnetic field. The experimental heat flux distributions agree fairly well with the theoretical ones.
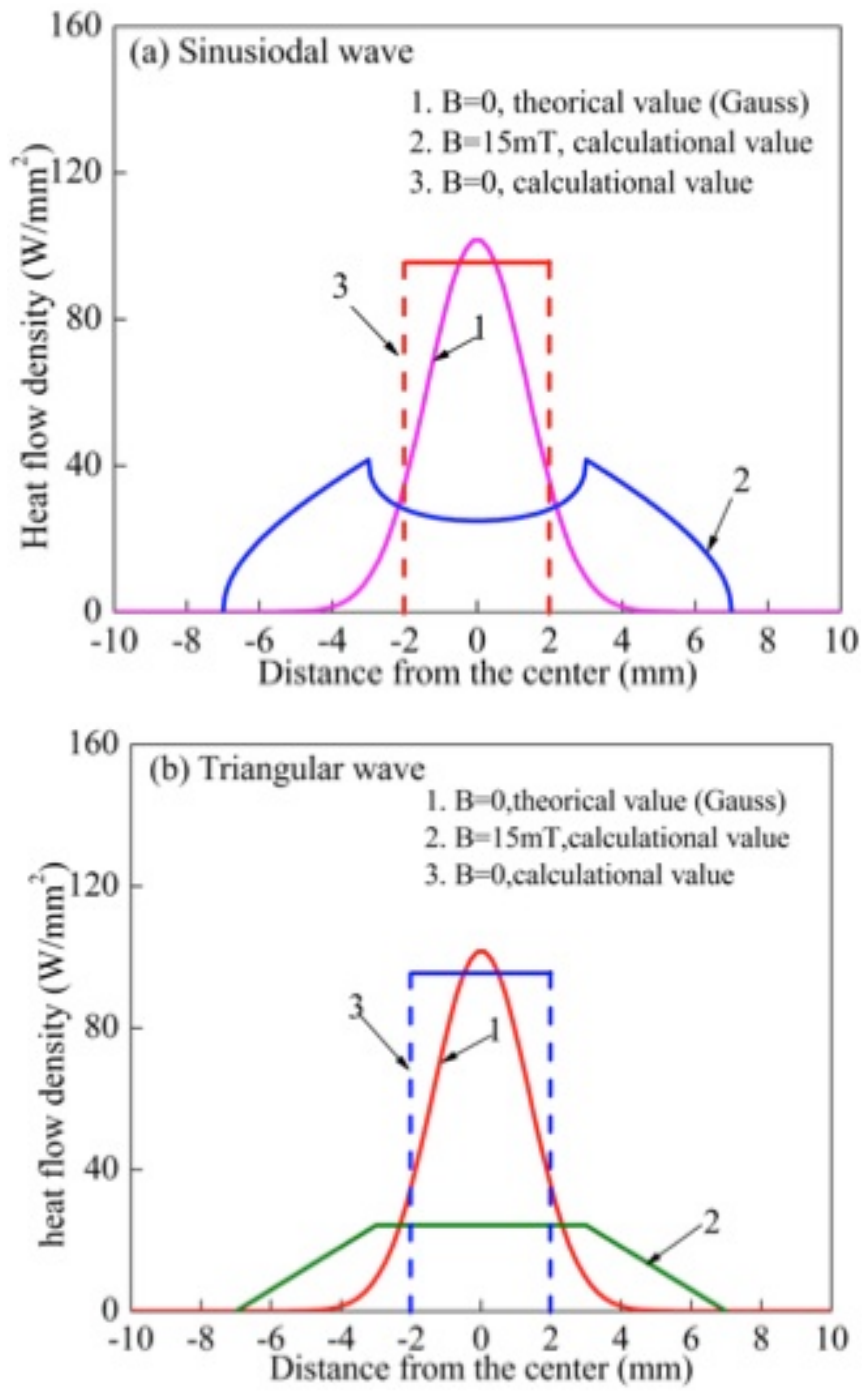

Fig. (7). Distributions of heat flux densities with or without alternating magnetic field.

\section{CONCLUSION}

This work has presented a new method to investigate the performance of a combined plasma arc under an external transverse magnetic field. With two models on the heat flux density distribution of combined plasma arc driven by the magnetic field with a sinusoidal current and a triangular current, the effects of process parameters such as the gas flow rate, arc current and overhang from the nozzle outlet to the anode workpiece on the characteristics of combined plasma arc are revealed. It is feasible to predict the forms and the heat flux density distributions of combined plasma arc under different operation conditions and to control the heating area and the heat flux density of combined plasma arc on the workpiece surface. Obtained results are summarized as follows:
It is efficient to control the heating area and to flatten the heat flux density distribution of combined plasma arc with an external transverse alternating magnetic field. Increasing the external magnetic flux density is to cause the radius of heat flux density distribution to increase, which results in the heat flux density at the center of arc root and its gradient near the center to decrease. However, an overly strong magnetic field makes the arc motion unstable.
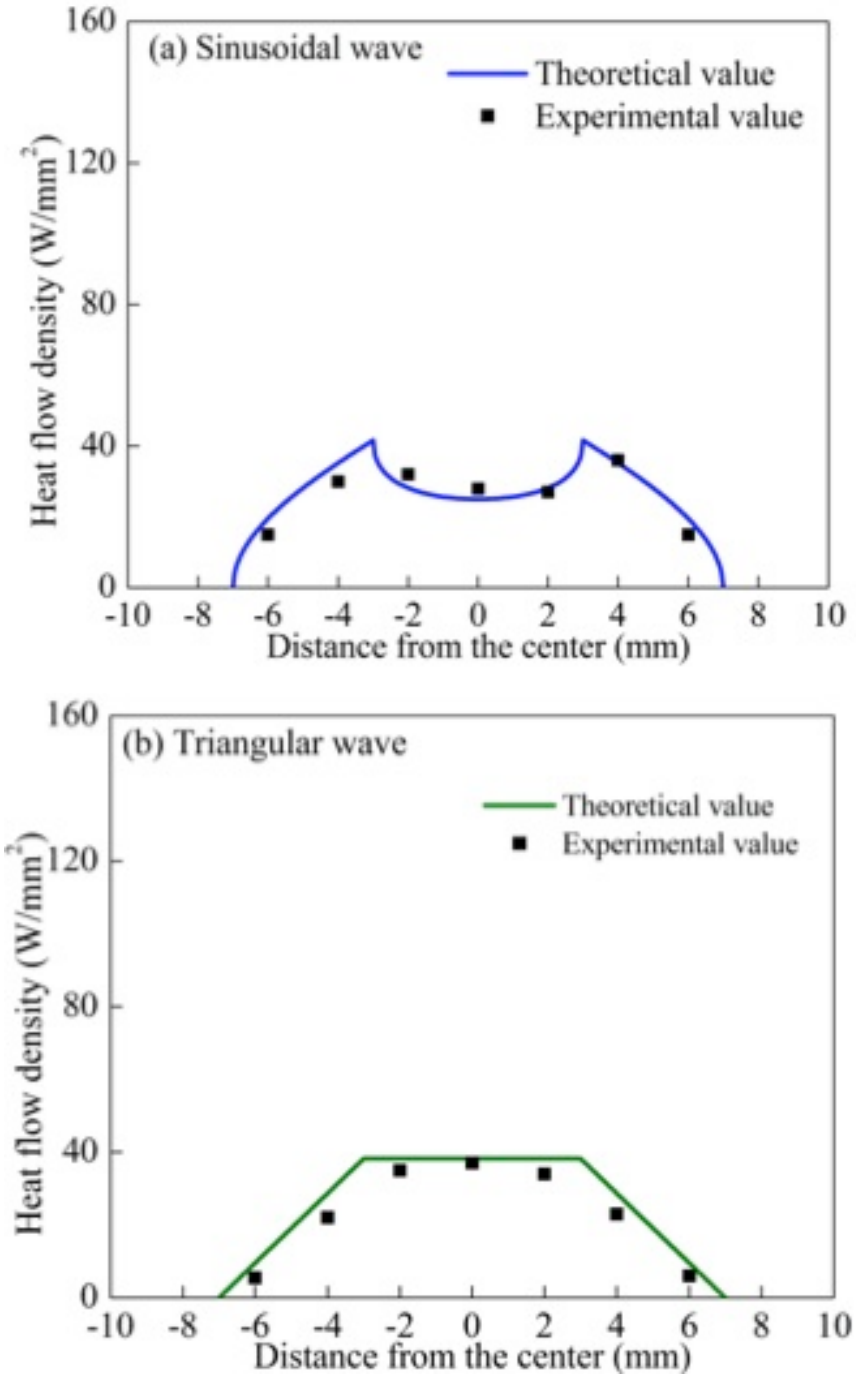

Fig. (8). Comparison of experimental results with theoretical ones of heat flux density.

Under the same external magnetic flux density, heat flux density distribution of combined plasma arc varies with the change of process parameters. Accordingly, the radius of heat flux distribution descends and the heat flux density at the center of the arc root increase. Meanwhile, increasing arc current or gas flow rate is to uniform the heat flux density of the whole heating region.

Increasing the overhang, the heat flux density at the center of arc root and its gradient near the center is to reduce. Consequently, it means that heightening the overhang from the nozzle outlet to the workpiece surface is to uniform the heat flux density on the entire heating area.

Calculated heat flux density distribution of the combined plasma arc driven by the external transverse-alternating 
magnetic field shows a good agreement with experimental data. Changing the waveform can control the heat flux density distribution. The magnetic field with triangular alternating current shows more uniform heat flux density distribution on the anode rather than sinusoidal one. This approach to get uniform heat flux density distribution on the anode surface will give an effective controllability to the combined plasma arc application.

\section{CONFLICT OF INTEREST}

The authors confirm that the content of the paper entails no conflict of interest.

\section{ACKNOWLEDGEMENTS}

The project was supported by the National Nature Science Foundation of China (Grant No. 51205237 and 51375284), also supported by Promotive research fund for excellent young and middle-aged scientists of Shandong Province (Grant No. BS2010ZZ009), respectively.

\section{REFERENCES}

[1] J.B. Meng, W.J. Xu, and W.Q. Song, "Modelling of reactive kinetics in the metal surface contaminant cleaning using atmospheric pressure plasma arc", Appl. Surf. Sci., vol. 254, pp. 6826-30, 2008.

[2] A.L. Lei, G.H. Li, L.J. Feng, and N. Dong, "Structure and abradability $\mathrm{Cu}-\mathrm{Al}_{2} \mathrm{O}_{3}$ gradient coatings fabricated by plasma spraying”, Trans. Chin. Weld. Institution, vol. 29, pp. 65-68, 2008.

[3] T. Yamamoto, K. Takeda, T. Toh, and J. Tanaka, "Distribution of heat flux transported by a magnetically diriven arc", Thin Solid Films, vol. 515, pp. 4228-33, 2007.
[4] Y.Y. Kiselev, and V.K. Pogora, "Radial distribution of the heatflux density in the bearing spot of a plasma cutting arc", J. Eng. Phy., vol. 59, pp. 1495-8, 1990.

[5] H.X. Wang, K. Cheng, X. Chen, and W.X. Pan, "Threedismensional modeling of heat transfer and fluid flow in laminar plasma material re-melting processing", Int. J. Heat. Mass. Tran., vol. 49, pp. 2254-64, 2005.

[6] J.B. Meng, and X.J. Dong, "Numerical simulation and analysis on jet characteristics of combined plasma arc", Open Mech. Eng. J., vol. 5, pp. 78-86, 2011.

[7] J. Awrejcewicz, "Combined analytical and numerical analysis of oscillations in the string-type generator", J. Appl. Math. Mech., vol. 74, pp. 432-4, 1994.

[8] J. Awrejcewicz, "Nonlinear oscillations of a string caused by the electromagnetic field", J. Tech. Phys., vol. 35, pp. 5-12, 1994.

[9] J. Awrejcewicz, and Z. Koruba, Classical Mechanics. Applied Mechanics and Mechatronics. Springer, New York, 2012.

[10] D.B. Sobyanin, B.G. Gavrilov, and I.M. Podgorny, "Laboratory investigation of plasma jet interaction with transverse magnetic field", Adv. Space Res., Vol. 29, pp. 1345-9, 2002.

[11] A. Zhainakov, R.M. Urusov, and T.E. Urusova, "Numerical simulation of gas dynamic flows formed by an electric arc in external transverse magnetic field", High Temp., Vol. 40, pp. 199203, 2002.

[12] R. Akiho, K. Takeda, M. Sugimoto, and Y. Noguchi, "Magnus effect on arc driven by alternating magnetic field in swirling plasma gas", ISIJ International, vol. 54, No. 4, pp. 774-80, 2014.

[13] W.J. Xu, Y.X. Guo, W.Q. Song, J.B. Meng, and Z.G. Li, "Experimental study of flexible formation using plasma arc controlled by alternate magnetic field", Mech. Sci. Technol., vol. 26, pp. 387-90, 2007.

[14] J.B. Meng, and X.J. Dong, "Mathematical modeling of heat flux distribution of plasma arc by transverse alternating magnetic field", Open Mech. Eng. J., vol. 7, pp.1-8, 2013.

(C) Dong et al.; Licensee Bentham Open.

This is an open access article licensed under the terms of the Creative Commons Attribution Non-Commercial License (http://creativecommons.org/licenses/ by-nc/3.0/) which permits unrestricted, non-commercial use, distribution and reproduction in any medium, provided the work is properly cited. 